\title{
Signatures of molecular correlations in few-electron dynamics of coupled quantum dots
}

\author{
Andrea Bertoni, ${ }^{1}$ Juan I. Climente, ${ }^{1}$ Massimo Rontani, ${ }^{1}$ Guido Goldoni, ${ }^{1,2}$ and Ulrich Hohenester ${ }^{3}$ \\ ${ }^{1}$ CNR-INFM-National Research Center on nano-Structures and bio-Systems at Surfaces (S3), Via Campi 213/A, 41100 Modena, Italy \\ ${ }^{2}$ Dipartimento di Fisica, Università di Modena e Reggio E., 41100 Modena, Italy \\ ${ }^{3}$ Institut für Physik, Karl-Franzens-Universität Graz, Universitätsplatz, 5, 8010 Graz, Austria
}

(Received 29 October 2007; published 21 December 2007)

\begin{abstract}
We study the effect of Coulomb interaction on the few-electron dynamics in coupled semiconductor quantum dots by exact diagonalization of the few-body Hamiltonian. The oscillation of carriers is strongly affected by the number of confined electrons and by the strength of the interdot correlations. Single-frequency oscillations are found for either uncorrelated or highly correlated states, while multifrequency oscillations take place in the intermediate regime. Moreover, Coulomb interaction renders few-particle oscillations sensitive to perturbations in spatial directions other than that of the tunneling, contrary to the single-particle case. The inclusion of acoustic phonon scattering does not modify the carrier dynamics substantially at short times, but it can damp oscillation modes selectively at long times.
\end{abstract}

DOI: 10.1103/PhysRevB.76.233303

PACS number(s): 73.21.La, 03.67.Lx, 72.10.Di

Low-dimensional heterostructures enable direct probing of the time evolution of carriers. In particular, charge oscillations between coupled quantum structures have been measured in a number of different systems, such as Josephson junctions, ${ }^{1}$ quantum wells, ${ }^{2}$ or quantum dots. ${ }^{3,4}$ Coupled quantum dots (CQDs), where the number of confined carriers can be controlled experimentally, are a most interesting case as they possess a discrete energy spectrum which stems from the quantum confinement in all three spatial directions, thus constituting the physical realization of the particle-in-the-box problem. Understanding charge oscillations in these structures is not only of fundamental importance but also of technological relevance, since control of the coherent dynamics of charge states is at the base of many proposals for novel nanoelectronic devices ${ }^{5-7}$ and quantum logic gates. ${ }^{8-10}$ This has motivated recent attempts to achieve control of charge localization and dynamics in CQD systems by means of microwave excitations, ${ }^{11}$ static, ${ }^{4}$ and time-dependent ${ }^{12,13}$ electric fields.

Coherent charge oscillations between CQDs have been recently demonstrated in the single-electron ${ }^{11}$ and few-electron ${ }^{3,4}$ regimes. The origin and behavior of these oscillations in the former case are well understood: when a single electron is placed in one of the dots of a CQD system, with no other electron in it, it oscillates back and forth between the dots with a frequency given by the energy difference between the bonding and antibonding "molecular" states. When a larger number of electrons are present, the system behavior is much less understood. Recent experiments working in the latter regime exposed a singlefrequency oscillation of the carriers, ${ }^{3,4}$ which was interpreted in terms of an effective single-electron picture. However, in general, one would expect more complicated oscillation patterns owing to the nontrival density of states of Coulombcorrelated few-body systems. ${ }^{13-16}$

In this Brief Report, we theoretically investigate charge oscillations in few-electron CQDs. We show that, as an effect of Coulomb correlations, the amplitude, period, and shape of these oscillations are strongly dependent on the number of electrons confined in the structure. Either single-frequency or multifrequency charge oscillations occur, depending on the strength of the correlations between molecular levels of the
CQD. Additional external magnetic fields are shown to provide a versatile means for tuning the period of the charge oscillations. This unique behavior due to the full spatial quantization is characteristic of quantum dots and clearly differs from the plasmon oscillations in coupled quantum wells. ${ }^{2,17}$ We also investigate the effect of the acoustic phonon bath and show that the different strengths of electronphonon interaction for different few-electron states lead to selective suppression of modes in the multifrequency dynamics.

We consider a system of two vertically coupled dots, as depicted in the first panel of Fig. 1, populated with $N$ interacting electrons. The dots are gated GaAs/AlGaAs heterostructures, as those built in Ref. 18. The conduction-band single-electron states are described within a threedimensional envelope function approximation, including an electric field $E_{z}$ along the $z$ direction. ${ }^{19}$ For this cylindrically symmetric configuration, the single-particle eigenfunctions can be given the separable form $\psi_{n m g s}(\mathbf{r}, \sigma)$ $=\phi_{n m}(x, y) \varphi_{g}(z) \chi_{s}(\sigma)$, with $n$ and $m$ radial and angular quantum numbers of the Fock-Darwin state, ${ }^{16} g=0,1$ labeling the bonding and antibonding eigenfunctions associated with the double-well potential, and $s$ the spin orientation. In order to compute the few-electron states exactly, we use a full configuration interaction approach: ${ }^{20}$ the single-particle states are populated with $N$ electrons in all possible ways to construct a basis of the Slater determinants $\left|\Phi_{j}\right\rangle$, where $j$ stands for the set of many-body quantum numbers. Then, the threedimensional $\mathrm{N}$-electron Hamiltonian is diagonalized. We first assume a closed system, so that coherent charge oscillations take place. In the last part of this Brief Report, we briefly investigate the effect of phonons.

Initially, the CQD is subject to an electrostatic bias $E_{z}$ which favors localization in the lower dot (QD2). Then, at time $t=0$, the bias is removed (nonadiabatically) and the carriers start oscillating between the two dots. In order to simulate this process, the configuration interaction calculation described above is performed twice: first, by taking $E_{z}>0$, then $E_{z}=0$. The computed eigenstates are $\left|\widetilde{\Psi}_{l}\right\rangle=\Sigma_{j} \widetilde{c}_{l j}\left|\widetilde{\Phi}_{j}\right\rangle$ and $\left|\Psi_{l}\right\rangle=\sum_{j} c_{l j}\left|\Phi_{j}\right\rangle$, with and without the $E_{z}$ field, respectively. We take, as the initial state of our few-particle system, the 

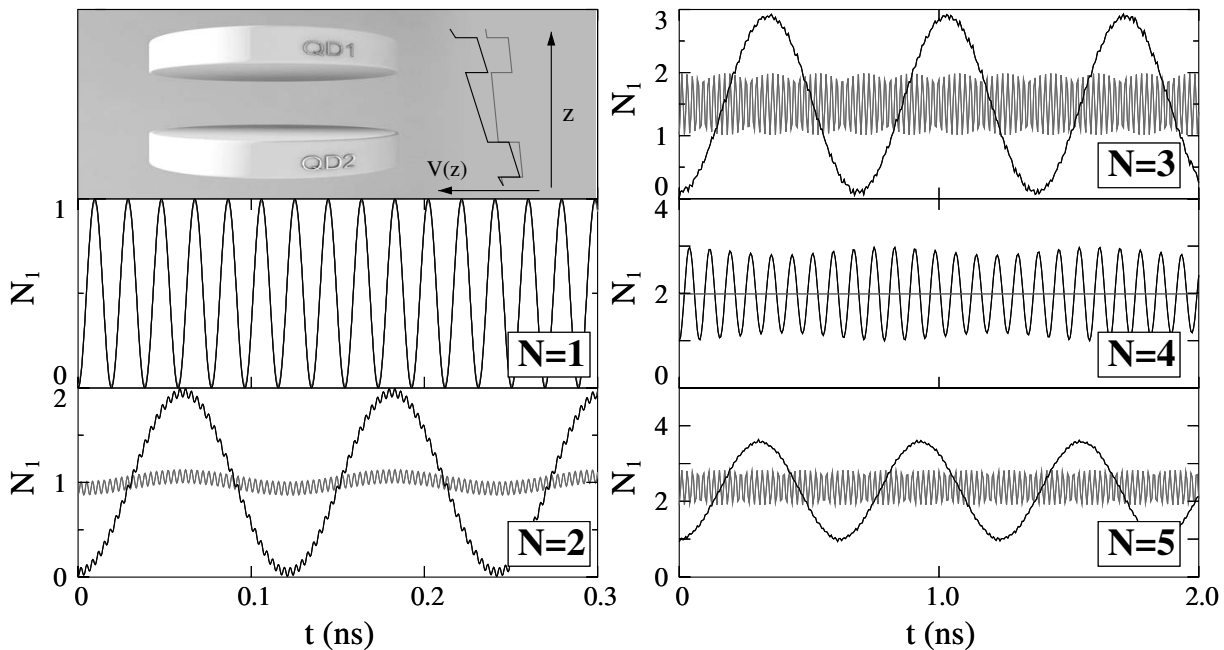

FIG. 1. Schematic of the CQD structure under study and occupation probability in the upper dot as a function of time for $\mathrm{N}$-electron systems. Gray and black lines represent the oscillation patterns for weak $\left(E_{z}=50 \mathrm{kV} / \mathrm{m}\right)$ and strong $\left(E_{z}=170 \mathrm{kV} / \mathrm{m}\right)$ initialization biases, respectively. The dots have thickness $W=10 \mathrm{~nm}$, lateral harmonic confinement $\hbar \omega_{0}=3 \mathrm{meV}$, and the interdot separation $L_{b}$ $=8 \mathrm{~nm}$. The prominent oscillation period depends mainly on the number of oscillating electrons (note the two different scales for the time). ground state in the biased condition $\left|\widetilde{\Psi}_{0}\right\rangle$. Its time evolution, after the removal of the bias, can be computed from

$$
\left|\widetilde{\Psi}_{0}(t)\right\rangle=\sum_{l} e^{-i / \hbar E_{l} t}\left\langle\Psi_{l} \mid \widetilde{\Psi}_{0}\right\rangle\left|\Psi_{l}\right\rangle
$$

Here, the sum runs over the $l$ unbiased states, whose energy is $E_{l}$, participating in the spectral decomposition of $\left|\widetilde{\Psi}_{0}\right\rangle$. In order to visualize the charge oscillations, we evaluate the particle density inside the upper dot (QD1) $N_{1}$. This magnitude has been resolved experimentally using pump-andprobe techniques $^{3}$ or single-electron transistors. ${ }^{4}$

In Fig. 1, we illustrate the time-dependent occupancy of QD1 for $N=1,2,3,4,5$. Gray and black lines represent weak and strong initialization biases (see caption), respectively, as schematically reported by the two $V(z)$ profiles in the first panel. For $N=1$, we retrieve the expected sinusoidal oscillation, whose frequency is given by the bonding-antibonding energy separation $\Delta E_{\mathrm{BAB}}$. Both weak and strong biases completely localize the electron in QD2, thus giving rise to essentially the same curve. However, as $N$ increases, the initialization bias starts playing a critical role, as it determines the number of electrons localized in each dot at time zero. This, in turn, gives rise to very different oscillation patterns (compare black and gray curves for $N>1$ ).

An inspection of the few-electron oscillations in Fig. 1 shows that they exhibit multiple frequencies with different amplitudes (most apparent in the $N=2$ panel). This is a signature of Coulomb interaction in the interdot dynamics, which can be understood from the coefficients of the spectral decomposition of the biased state $\left\langle\Psi_{l} \mid \widetilde{\Psi}_{0}\right\rangle$ : only the unbiased states which have a finite overlap will contribute to the time evolution in Eq. (1). In the single-electron case, the in-plane and vertical degrees of freedom decouple. The initial state is localized in QD2 and results to be the linear combination of one bonding and one antibonding unbiased states. This is because the in-plane $x y$ component of the wave function is the same with and without bias, while, in $z$ direction, the ground biased state is given by $\varphi_{0}(z)+\varphi_{1}(z)$. Therefore, only these two states contribute to the spectral decomposition, and the oscillation frequency is given by their energy difference. By contrast, in the few-electron case, Coulomb interaction mixes the radial and vertical degrees of freedom, so that the in-plane parts of the wave functions with and without $E_{z}$ are no longer identical. As a result, the spectral decomposition may involve several pairs of bonding-antibonding states with different radial wave functions. Each pair contributes to the time evolution with its own frequency, given by its bondingantibonding energy splitting, and an amplitude that is proportional to its spectral decomposition weight.

The mixing of radial and vertical degrees of freedom due to Coulomb interaction renders charge oscillations sensitive to perturbations in the $x y$ plane, even though they do not affect the single-particle tunneling. This is shown in Fig. 2, where we compare single- and few-electron oscillations in the presence and the absence of a magnetic field applied along $z$. While the $N=1$ oscillation is insensitive to the field, the frequencies of the $N=2$ and $N=3$ ones are increased. This signature of electron-electron interaction could be used in experiments to distinguish between the dynamics of independent and correlated electrons. It also offers a unique way to modulate the few-particle oscillation frequency.

To gain further insight into the role of Coulomb interaction, in Fig. 3 we compare the oscillation patterns in a CQD

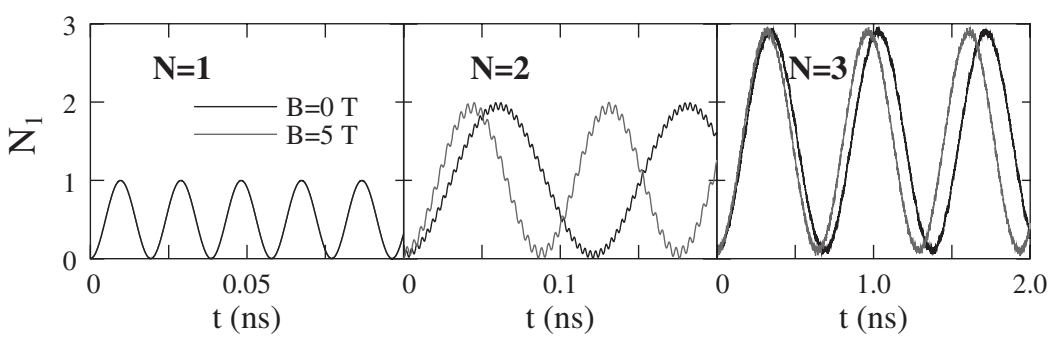

$233303-2$
FIG. 2. Occupation probability in the upper dot as a function of time for $N$-electron systems. Gray and black lines represent the oscillation patterns with $(B=5 \mathrm{~T})$ and without $(B=0 \mathrm{~T})$ magnetic field, respectively. The CQD structure and biases $E_{z}$ are the same as in Fig. 1. 

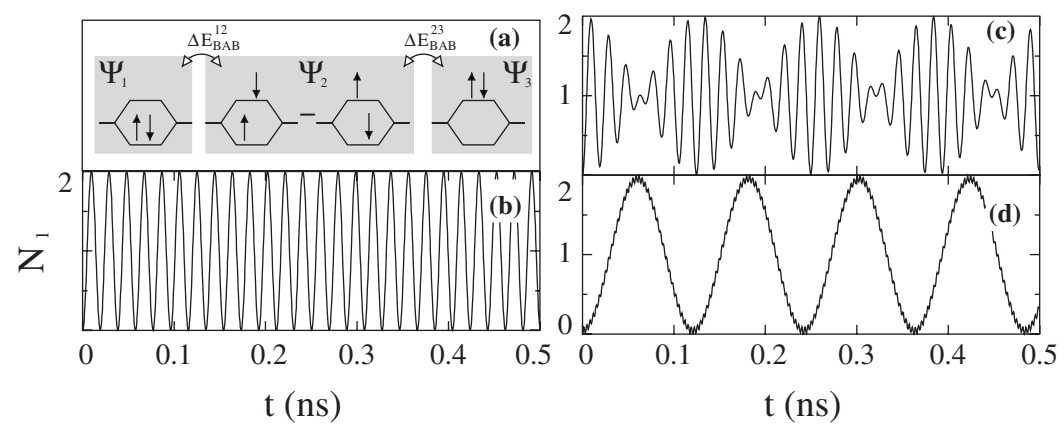

FIG. 3. (a) Main unbiased states contributing to the spectral decomposition of the ground state in the $N=2$ CQD of Fig. 1 at $E_{z}=150 \mathrm{kV} / \mathrm{m}$. Occupation probability of the upper dot in systems with (b) null $\left(\epsilon^{*}=\infty\right)$, (c) partially quenched $\left(\epsilon^{*}\right.$ $\left.=10^{3}\right)$, and $(\mathrm{d})$ regular $\left(\epsilon^{*}=12.9\right)$ Coulomb interactions. with no interaction (panel b), artificially quenched interaction (panel c), and regular interaction (panel d). In all cases, the electric field initializes both electrons in QD2, and yet major changes take place as the Coulomb intensity is varied. Again, the changes can be understood by analyzing the main unbiased states contributing to the spectral decomposition of the biased state. These are the $s$-shell singlets, $\Psi_{1}, \Psi_{2}$, and $\Psi_{3}$, represented in Fig. 3(a) by their dominating electronic configuration. In the schematic representation, electrons are indicated by arrows, whose direction is the spin state, and can occupy the single-particle bonding (lower horizontal line) or antibonding (upper horizontal line) states. For vanishing Coulomb interaction, $\widetilde{\Psi}_{0}$ is composed with equal weight of the two bonding-antibonding pairs $\left(\Psi_{1}+\Psi_{2}\right)$ and $\left(\Psi_{2}+\Psi_{3}\right)$. The frequency arising from the two pairs is identical, $\Delta E_{\mathrm{BAB}}^{12}=\Delta E_{\mathrm{BAB}}^{23}=E_{t}\left(E_{t}\right.$ is the single-particle tunneling energy), and the oscillation shown in Fig. 3(b) is reminiscent of a single-particle one. Switching on a weak Coulomb perturbation [Fig. 3(c)] introduces a small departure from this limit, here, $\Delta E_{\mathrm{BAB}}^{12}=0.24 \mathrm{meV}$ and $\Delta E_{\mathrm{BAB}}^{23}=0.20 \mathrm{meV}$ : now, the two pairs have similar amplitudes but different energies. The superposition of the two harmonic motions gives rise to a beat with modulated amplitude and frequency. Finally, for a realistic Coulomb interaction [Fig. 3(d)], $\Delta E_{\mathrm{BAB}}^{12}$ $=1.26 \mathrm{meV}$ and $\Delta E_{\mathrm{BAB}}^{23}=0.04 \mathrm{meV}$, the weights of the two bonding-antibonding pairs are very different. As a consequence, there is one dominating oscillation mode with large amplitude and low frequency and another with small amplitude and high frequency.

The sizable changes of the bonding-antibonding energy splittings in the presence of Coulomb interaction are an effect of the electronic correlation between molecular states, often disregarded in previous studies of multiparticle dynamics in coupled quantum dots ${ }^{13,14}$ and wells. ${ }^{17}$ The weaker the interdot correlation, the stronger the multifrequency character and vice versa. Therefore, one can control the nature of the charge oscillations by designing CQD structures in the regimes of either weak or strong interdot correlation with respect to $E_{t}$. This is shown in Fig. 4, where we compare the $N=2$ and $N=3$ charge oscillations for different structural parameters, keeping a realistic value for the Coulomb interaction throughout. In (a), the interdot barrier is thin and hence the tunneling energy is large. The large splitting between molecular orbitals implies weak interdot correlation, which leads to an oscillation pattern resembling the multifrequency beat of Fig. 3(c). In (b), the radial confinement is increased, which increases the Coulomb repulsion within the structure. Since the vertical confinement is unchanged, interdot correlation moves into the strong regime and a quasisinglefrequency behavior, similar to that of Fig. 3(d), is retrieved. In (c), the barrier is made thicker with respect to (a), so that the tunneling energy diminishes. This again enhances the interdot correlation, leading to the quasisingle-frequency behavior. We point out that the small tunneling energy could be the reason for the single-frequency oscillations reported in Refs. 3 and 4 experiments.

The electron dynamics in CQDs is severely affected by dissipative processes. ${ }^{21}$ In what follows, we shall investigate which effects may appear in the few-electron charge oscillations due to the interaction with the $20 \mathrm{mK}$ acoustic (deformation-potential and piezoelectric) phonon bath. We calculate the transition rate $\gamma_{l i}$ between the correlated states $\left|\Psi_{l}\right\rangle$ and $\left|\Psi_{i}\right\rangle$ according to Ref. 22 and employ the Pauli master equation to evaluate the system's time evolution: $\frac{d \rho_{i j}}{d t}=\frac{i}{\hbar}\left(E_{j}-E_{i}\right) \rho_{i j}-\Sigma_{l} \frac{\gamma_{l j}+\gamma_{l i}}{2} \rho_{i j}+\delta_{i j} \sum_{l} \gamma_{i l} \rho_{l l}$. We stress that, here, (a)

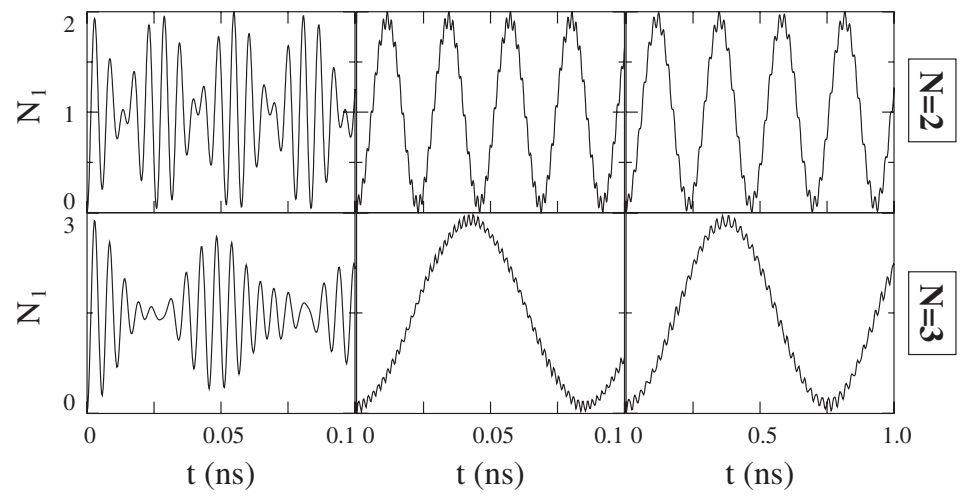

FIG. 4. Occupation probability in the upper dot as a function of time for $N=2$ (upper row) and $N=3$ (lower row). Here, $E_{z}=500 \mathrm{kV} / \mathrm{m}$ and $W=10 \mathrm{~nm}$. The lateral confinement and interdot barrier thickness in each column are (a) $\hbar \omega_{0}$ $=1 \mathrm{meV}$ and $L_{b}=6 \mathrm{~nm}$, (b) $\hbar \omega_{0}=6 \mathrm{meV}$ and $L_{b}$ $=6 \mathrm{~nm}$, and (c) $\hbar \omega_{0}=1 \mathrm{meV}$ and $L_{b}=10 \mathrm{~nm}$. Moreover, $\epsilon^{*}=12.9$ in all cases. 

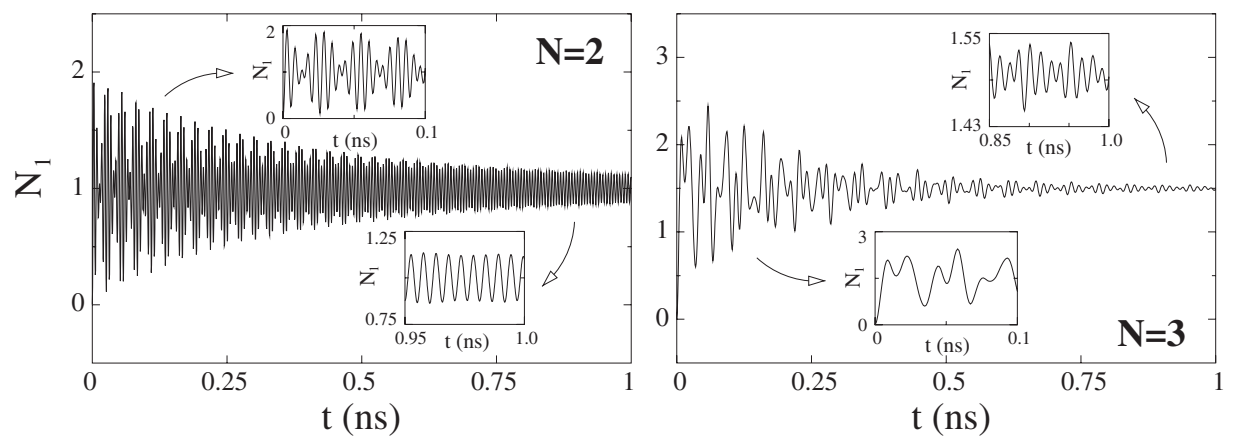

FIG. 5. Occupation probability as a function of time in $N=2$ and $N=3$ CQDs subject to phonon interaction. The insets zoom in at short and long times. Note that the $N=2$ case evolves into a singlefrequency oscillation. The CQDs have $\hbar \omega_{0}, W=10 \mathrm{~nm}, L_{b}=6 \mathrm{~nm}$ $(N=2)$, and $L_{b}=8 \mathrm{~nm}(N=3)$ and are initialized with a bias $E_{z}$ $=500 \mathrm{kV} / \mathrm{m}$. $\rho_{i j}$ is an element of the density matrix corresponding to the (unbiased) few-particle states, with energies $E_{i}$ and $E_{j}$.

Figure 5 shows the resulting charge oscillations for a CQD with $N=2$ (left panel) and $N=3$ (right panel). In both cases, the oscillation amplitude is clearly damped by the phonons. However, since the transition rate is different for each couple of initial and final states, the different modes contributing to the multifrequency oscillations are damped at different rates. As a consequence, in some CQD structures, all but one mode are quickly suppressed, and the initially multifrequency oscillation turns into a single-frequency one at latter times (compare the insets in the $N=2$ panel for short and long $t$ ). For $N>2$, a higher number of correlated states participate in the spectral decomposition of the biased state, so that more oscillation modes show up in the charge oscillation. As a result, it is difficult to find conditions where only one mode survives the phonon damping (see insets of $N=3$ panel). Therefore, this is unlikely to be responsible for the single-frequency oscillations observed in Refs. 3 and 4.
In summary, we have shown that the few-electron dynamics of CQDs is strongly affected by interdot electronic correlations. Drastic changes in the oscillation pattern, from simple sinusoidal to complicated beats, take place depending on the correlation strength. Electronic interaction further renders few-electron dynamics very sensitive to perturbations in all directions of the space and not only in that of the tunneling. Upon inclusion of phonon damping, few-electron charge oscillations may experience an additional effect, namely, a selective suppression of frequency modes. This is, however, unlikely to be responsible for the single-particle-like oscillations reported in early experiments, ${ }^{3,4}$ which can be understood as an effect of strong molecular correlation, due to the small tunneling energy.

We thank E. Molinari and F. Troiani for most helpful discussions. This work has been supported by projects FIRB No. RBIN04EY74 and INFM-Cineca Calcolo Parallelo 2007. J.I.C. acknowledges support from Marie Curie IEF under Project No. MEIF-CT-2006-023797.
${ }^{1}$ A. Pashkin, T. Yamamoto, O. Astafiev, Y. Nakamura, D. V. Averin, and J. S. Tsai, Nature (London) 421, 823 (2003).

${ }^{2}$ K. Leo, J. Shah, E. O. Göbel, T. C. Damen, S. Schmitt-Rink, W. Schäfer, and K. Köhler, Phys. Rev. Lett. 66, 201 (1991).

${ }^{3}$ T. Hayashi, T. Fujisawa, H. D. Cheong, Y. H. Jeong, and Y. Hirayama, Phys. Rev. Lett. 91, 226804 (2003); T. Fujisawa, T. Hayashi, H. D. Cheong, Y. H. Jeong, and Y. Hirayama, Physica E (Amsterdam) 21, 1046 (2004).

${ }^{4}$ J. Gorman, D. G. Hasko, and D. A. Williams, Phys. Rev. Lett. 95, 090502 (2005).

${ }^{5}$ C. Lent, P. D. Tougaw, and W. Porod, Appl. Phys. Lett. 62, 714 (1993)

${ }^{6}$ O. Astafiev, S. Komiyama, and T. Kutsuwa, Appl. Phys. Lett. 79, 1199 (2001).

${ }^{7}$ O. Gywat, G. Burkard, and D. Loss, Phys. Rev. B 65, 205329 (2002).

${ }^{8}$ L. Fedichkin, M. Yanchenko, and K. A. Valiev, Nanotechnology 11, 387 (2000).

${ }^{9}$ W. G. van der Wiel, T. Fujisawa, S. Tarucha, and L. P. Kouwenhoven, Jpn. J. Appl. Phys., Part 1 40, 2100 (2001).

${ }^{10}$ U. Hohenester, Phys. Rev. B 74, 161307(R) (2006); J. Fabian and U. Hohenester, ibid. 72, 201304(R) (2005).

${ }^{11}$ J. R. Petta, A. C. Johnson, C. M. Marcus, M. P. Hanson, and A. C. Gossard, Phys. Rev. Lett. 93, 186802 (2004).

${ }^{12}$ P. I. Tamborenea and H. Metiu, Europhys. Lett. 53, 776 (2001);
G. E. Murgida, D. A. Wisniacki, and P. I. Tamborenea, Phys. Rev. Lett. 99, 036806 (2007).

${ }^{13}$ P. Zhang and X. G. Zhao, Phys. Lett. A 271, 419 (2000).

${ }^{14}$ Z. Y. Zhang and S. J. Xiong, Phys. Rev. E 57, 3668 (1998).

${ }^{15}$ T. Chakraborty, Quantum Dots (North-Holland, Amsterdam, 1999).

${ }^{16}$ L. Jacak, P. Hawrylak, and A. Wojs, Quantum Dots (Springer, Berlin, 1998).

${ }^{17}$ O. E. Raichev, Phys. Rev. B 51, 17713 (1995).

${ }^{18}$ D. G. Austing, T. Honda, K. Muraki, Y. Tokura, and S. Tarucha, Physica B 249-251, 206 (1998).

${ }^{19}$ The confining potential is $V(\mathbf{r})=V(z)+E_{z} z+\frac{1}{2} m^{*} \omega_{0}^{2}\left(x^{2}+y^{2}\right)$. Here, $V(z)$ represents the symmetric double well given by the band offset of the materials, $E_{z}$ is an electric field, and the last term gives the parabolic confinement in the $x y$ plane. We use the following GaAs/AlGaAs material parameters: effective mass $m^{*}=0.067$, band offset $V_{c}=0.243 \mathrm{eV}$, and effective dielectric constant $\epsilon^{*}=12.9$ (unless otherwise stated).

${ }^{20}$ M. Rontani, C. Cavazzoni, D. Bellucci, and G. Goldoni, J. Chem. Phys. 124, 124102 (2006); www.s3.infm.it/donrodrigo

${ }^{21}$ T. Fujisawa, T. Hayashi, and Y. Hirayama, J. Vac. Sci. Technol. B 22, 2035 (2004).

22 J. I. Climente, A. Bertoni, M. Rontani, G. Goldoni, and E. Molinari, Phys. Rev. B 74, 125303 (2006). 